${ }^{1}$ Centro de Información Toxicológica, Facultad de Medicina, Pontificia Universidad Católica de Chile. Santiago, Chile.

${ }^{2}$ Departamento de Salud Pública, Escuela de Medicina, Pontificia Universidad Católica de Chile. Santiago, Chile.

${ }^{3}$ Advanced Center for Chronic Diseases (ACCDiS). Fondo de Financiamiento de Centros de Investigación en Áreas Prioritarias (FONDAP). Santiago, Chile. ${ }^{4}$ Centro de Desarrollo Urbano Sustentable (Cedeus). Fondo de Financiamiento de Centros de Investigación en Áreas Prioritarias (FONDAP), Chile.

${ }^{5}$ School of Environment, University of Auckland, New Zealand.

${ }^{a}$ Estadístico. Doctor en Estadística.

${ }^{b}$ Geógrafo. Magíster en Geografía y Geomática. PhDc Geografía. 'Geógrafa. Magíster en Salud Pública. ${ }^{d}$ Enfermera. Diploma en Adulto Mayor. Magíster en Enfermería. Magíster en Epidemiología.

eEnfermero. Magíster en Salud Pública.

${ }^{f}$ Médico Veterinario. Magíster en Cinecias Biológicas, mención Ciencias Ambientales. Doctora en Salud Pública.

Los autores declaran no tener conflictos de interés. Este estudio fue financiado por el Gobierno Regional de Antofagasta a través

del Proyecto "Investigación Estudio Polimetales y Perfil Epidemiológico en Habitantes de Antofagasta" Código BIP $N^{\circ} 30462238-0$.

Recibido el 14 de septiembre de 2019 , aceptado el 28 de mayo de 2020.

Correspondencia a: Sandra Cortés Departamento de Salud Pública, Escuela de Medicina, Pontificia Universidad Católica de Chile. Santiago, Chile. scortesn@uc.cl

\section{Estudio de metales urinarios y plomo en sangre: parámetros poblacionales en Antofagasta, 2018}

\author{
JUAN CARLOS RÍOS ${ }^{1}$, LUIS VILLARROEL ${ }^{2, a}$, \\ MARISA TORRES ${ }^{2}$, JUAN PABLO ASTABURUAGA ${ }^{5, b}$, \\ CINTHYA LEIVA ${ }^{2, \mathrm{c}}$, PAZ COOK ${ }^{2,3, \mathrm{~d}}$, \\ PATRICIO MEDEL ${ }^{1, \mathrm{e}}$, SANDRA CORTÉS ${ }^{2,3,4, \mathrm{f}}$
}

\section{Urinary heavy metals and blood lead levels among residents of Antofagasta, Chile}

Background: The presence of toxic metals in human populations is strongly associated with chronic diseases. Aim: To determine levels of lead, chromium, cadmium, mercury and inorganic arsenic (AsIn) in the general population aged over 5 years in Antofagasta, Chile. Material and Methods: People living in Urban Antofagasta for at least five years were considered eligible. Biological samples were obtained to measure heavy metals. Results: One thousand two hundred three participants with a median age of 43 years (656 women) were studied. Their mean time of residence in the city was 30 years, and 52\% smoked. Eight percent of the adult population and $12 \%$ of children had AsIn values above $35 \mu \mathrm{g} / \mathrm{L}$, while $75 \%$ of the population had levels below $21.9 \mu \mathrm{g} / \mathrm{L}$. The other metals were below the risk levels defined by the health authority $(10 \mu \mathrm{g} / \mathrm{L}$ for chromium, 10 $\mu \mathrm{g} / \mathrm{L}$ for mercury, $2 \mu \mathrm{g} / \mathrm{L}$ for cadmium, 5 and $10 \mu \mathrm{g} / \mathrm{dL}$ for blood lead for children and adults, respectively). The factors associated with high levels of AsIn in adults were male sex, living more than 200 meters from monitoring points, and low schooling. In children, the associated variables were high intake of seafood products and having a caregiver with less than 8 years of schooling. Contrary to expectations, the greatest risk of presenting altered levels of the metals occurred in the population living more than 500 meters from the identified risk sources (90\% of the population). Conclusions: The findings of this study suggest that all potential sources of exposure to AsIn should be evaluated, and surveillance actions should be established to reduce involuntary exposure to this metalloid.

(Rev Med Chile 2020; 148: 746-754)

Key words: Arsenicals; Chile; Lead; Metals, Heavy; Urine.
L a Región de Antofagasta se localiza en el norte de Chile, entre los paralelos $21^{\circ} 28^{\prime}$ hasta aproximadamente los $25^{\circ} 55^{\prime}$ de latitud sur $^{1}$. Su capital regional es la ciudad de Antofagasta, zona de puerto marítimo y explota- ción minera, actividad que constituye su principal fuente productiva y económica. Dicha actividad ha tenido consecuencias en la salud de las personas y en el medio ambiente, a raíz de la generación de eventos de sobreexposición ambiental como el 
hidroarsenisismo acontecido en el período 19581970. Además, destacan el acopio de concentrados de plomo en zonas urbanas, problema acentuado por el aluvión ocurrido en 1991, y los depósitos de concentrados de plomo y zinc en el puerto de Antofagasta. Un estudio realizado en 2014 por la Secretaria Regional de Salud (SEREMI) de Salud encontró arsénico inorgánico, plomo y cadmio, en concentraciones más elevadas frente a instalaciones portuarias, en comparación con otros puntos del borde costero. Sin embargo, estos estudios no permiten asociar en una misma población, zona geográfica y período de tiempo, las mediciones realizadas en matrices ambientales, con los datos epidemiológicos. Por esta razón, se realizó un estudio que identifique con exactitud la magnitud de la exposición a metales pesados en la población de la región, especialmente en grupos de riesgo.

\section{Objetivo General}

Conocer la prevalencia y perfil epidemiológico de personas expuestas a plomo $(\mathrm{Pb})$, cadmio $(\mathrm{Cd})$, cromo $(\mathrm{Cr})$, mercurio $(\mathrm{Hg})$ y arsénico inorgánico (AsIn) que hayan tenido residencia ininterrumpida de 5 años o más en la ciudad de Antofagasta.

\section{Material y Métodos}

Se realizó un estudio epidemiológico-ambiental transversal, en una muestra de población urbana mayor de 5 años, residente en viviendas particulares de Antofagasta, que hubiesen vivido de forma ininterrumpida en la ciudad por al menos 5 años. Se excluyó a personas en condiciones de autonomía limitada, personas institucionalizadas, y a quienes no accedieran a entregar muestras biológicas. La muestra fue representativa de la población y se distribuyó de manera uniforme (Figura 1).

Se recopiló información sobre las siguientes variables: sociodemográficas, antecedentes de salud, probables fuentes de exposición a metales/ metaloides, e información de residencia en áreas de riesgo. Se tomaron muestras biológicas para medir arsénico inorgánico, cadmio, cromo y mercurio en orina, además de plomo en sangre. Los metales fueron medidos mediante la técnica de espectometría de masas con plasma inductivamente acoplado (ICP-MS) en el laboratorio de la Dirección de Investigaciones Científicas y Tecnológicas de la Pontificia Universidad Católica de Chile (DITUC).

El diseño muestral fue probabilístico, estratificado geográficamente, trietápico, de conglomerados y con distribución no proporcional de las unidades entre estratos. La estratificación geográfica se realizó a partir de la identificación de zonas de exposición de la población, donde se determinaron zonas con fuentes de contaminación fija y móviles (Figura 1), como es el caso de las fuentes emisoras de metales ubicadas en la zona del puerto, ex vertedero La Chimba, ferrocarril, las vías de mayor flujo vehicular y aquellas fuentes que emitan metales identificadas por el Servicio de Evaluación Ambiental (SEA). Además, se agregaron los puntos de monitoreo ambiental realizados por las SEREMI de Salud (2014-2015) y Medio Ambiente (2015-2016). En relación a lo anterior, se categorizó la muestra entre personas potencialmente expuestas y no expuestas a fuentes de contaminación ambiental.

El cálculo del tamaño muestral se realizó asumiendo una prevalencia de niveles mayores a 5 $\mathrm{dL}^{-1}$ de plomo en sangre con un margen de error de 0,4 puntos porcentuales, y una confianza de $95 \%$. Se consideró una pérdida de seguimiento de $20 \%$, con lo que se estableció una muestra de 1.280 personas.

\section{Recolección de datos}

Se diseñó una encuesta para recopilar datos epidemiológicos de la población en estudio. Este instrumento, se elaboró en base a encuestas nacionales previamente validadas. Posteriormente, fue aplicado en terreno por enfermeras capacitadas.

La toma de muestras biológicas se realizó en el hogar de cada participante, una vez firmado el consentimiento o asentimiento informado, según correspondiera en función de la edad.

\section{Análisis de datos}

El registro y captura de datos se realizó con la aplicación REDCap ${ }^{\circledR 2}$.

El acceso a la base de datos e informe final del estudio se realizó desde el Gobierno Regional de Antofagasta, a través del sistema de transparencia.

El análisis se realizó utilizando los programas SPSS versión 17.0 y SAS versión 9.4.

El plan de análisis consideró factores de ex- 


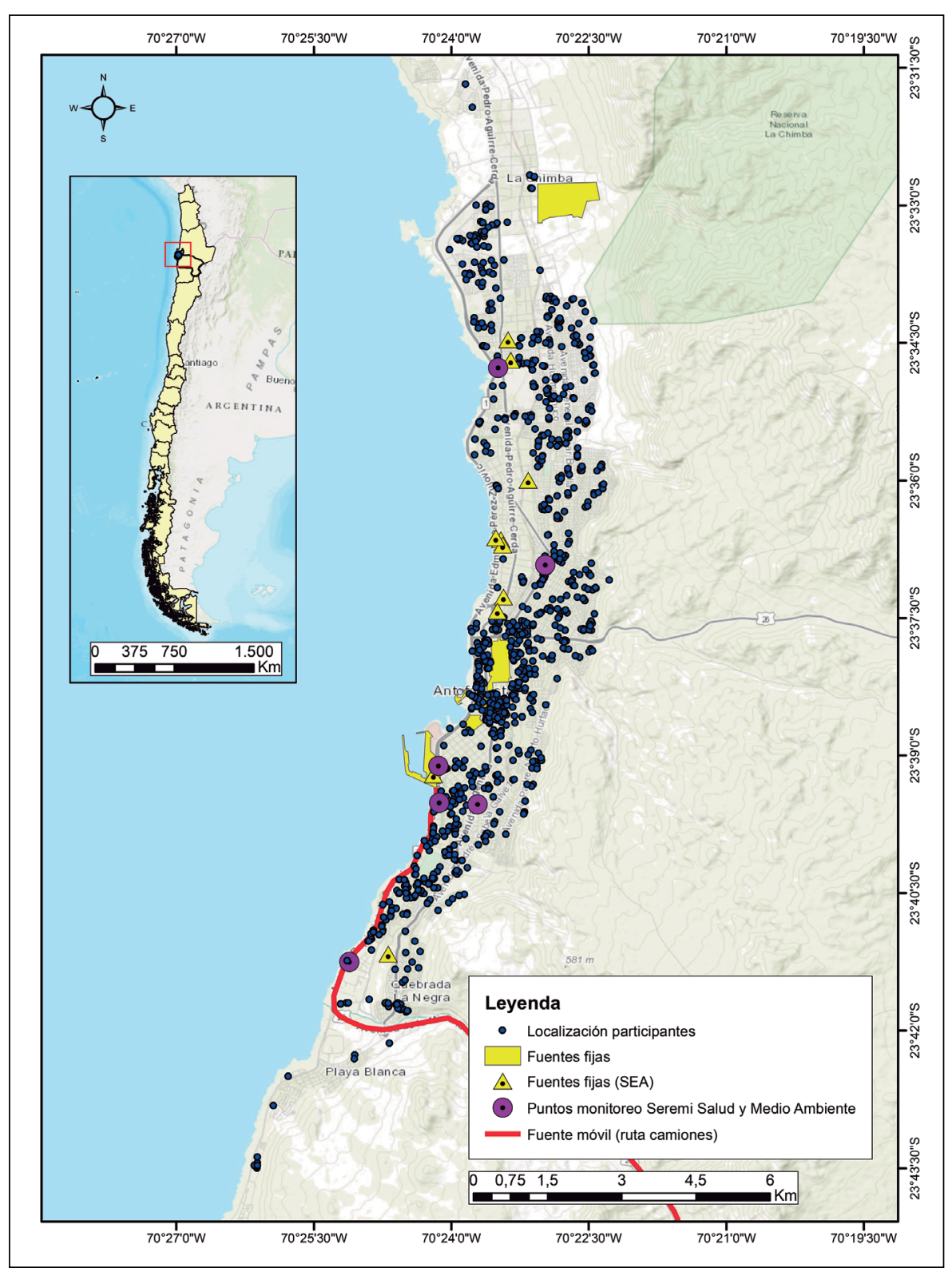

Figura 1. Localización participantes ciudad de Antofagasta. pansión, imputación de límites de detección para metales, estadísticas descriptivas, bivariadas y modelos multifactoriales.

Se consideró significativo los valores $p$ inferiores o iguales a 0,05 y se utilizó un intervalo de confianza de $95 \%$.

El estudio fue aprobado por el Comité de Ética Científico de la Facultad de Medicina de la Pontificia Universidad Católica.

\section{Resultados}

Para la muestra de adultos de la comuna de Antofagasta, el promedio de edad fue 42,7 años, sin diferencias estadísticamente significativas según sexo. Un bajo porcentaje de la muestra refirió tener solo estudios básicos $(14,7 \%)$, sin embargo, se observó un nivel de escolaridad significativamente mayor en hombres que en mujeres 
$(\mathrm{p}=0,01)$. En su mayoría, los participantes estaban adscritos al sistema público de salud $(66,1 \%)$, con un número significativamente mayor de hombres que pertenecía al sistema privado (Tabla 1). El mayor porcentaje de la muestra declaró ser chileno $(92,4 \%)$. Respecto al tiempo de residencia en la ciudad, promedió 30 años, tanto para hombres como para mujeres.

En relación a los estilos de vida, destacó el alto porcentaje de tabaquismo, sin diferencias por sexo (Tabla 1).

En cuanto a la exposición a metales descrita por los participantes del estudio, se encontró que variaba entre $3,3 \%(n=20)$ a níquel y $29,1 \%$ $(\mathrm{n}=184)$ a sílice, con diferencias significativas por sexo en la mayoría de ellos, excepto en la exposición a arsénico y cadmio, en los cuales la diferencia no alcanzó significancia estadística. Destaca que $22,6 \%$ de los participantes $(n=168)$ reportaron haber trabajado en fundición o minería, con predominio en hombres $(32,9 \%, \mathrm{p}<0,001)$ (Tabla 1$)$.

Es relevante señalar que, para la mayoría de los metales estudiados, se observó que las concentraciones fueron mayores en personas que vivían a más de 500 metros de las fuentes de contaminación y puntos de monitoreo, zona donde residía más de $90 \%$ de la población. Ejemplo de ello fue la concentración de arsénico en personas que vivían a menos de 200 metros de un punto de monitoreo ambiental, la cual alcanzó una media de $8,4 \mu \mathrm{g} / \mathrm{L}$, mientras que los que vivían a más de 500 metros presentaron un nivel de $17,2 \mu \mathrm{g} / \mathrm{L}$, diferencia que es estadísticamente significativa $(\mathrm{p}=0,001)$. La distribución de las concentraciones de los metales y arsénico evaluados en las poblaciones de adultos y niños, utilizando el percentil 50, 75 y 90 se evidencian en la Tabla 2. Destacan los mayores valores registrados para arsénico inorgánico en hombres, así como los altos valores de este metaloide en niños.

Al evaluar la prevalencia de exposición tomando como punto de corte los valores referenciales establecidos por el Ministerio de Salud ${ }^{3}$, destaca que $8 \%$ de los adultos y $12,9 \%$ de los niños y adolescentes tenían niveles de AsIn mayor a 35 $\mu \mathrm{g} / \mathrm{L}$, sin diferencias según sexo. Sin embargo, al revisar su distribución por percentiles, al menos el $75 \%$ de la población tenía niveles menores a 21,9 $\mu \mathrm{g} / \mathrm{L}$, lo que indica que un grupo reducido tenía niveles muy altos. Esta mayor concentración, tanto para adultos como para niños fue alcanzada en el percentil 95 (Figura 2).

Los niveles de los demás metales, incluyendo el plomo en sangre, se encontraron por debajo de las recomendaciones nacionales e internacionales, siendo menores en niños. Por esta razón, los resultados mostrados a continuación se focalizarán en la población con niveles de arsénico elevados.

Tabla 1. Características sociodemográficas de adultos y niños ciudad de Antofagasta, año 2018

\begin{tabular}{|c|c|c|c|c|c|c|}
\hline \multirow{2}{*}{$\begin{array}{l}\text { Características sociodemográficas } \\
\text { adultos }\end{array}$} & \multicolumn{2}{|c|}{ Total $(n=1.203)$} & \multicolumn{2}{|c|}{ Mujeres (n = 656) } & \multicolumn{2}{|c|}{ Hombres $(n=547)$} \\
\hline & Media o \% & $\mathbf{n}$ & Media o \% & $\mathbf{n}$ & Media o \% & $\mathbf{n}$ \\
\hline Edad, años (media) & 42,7 & 1.205 & 43,6 & 655 & 41,9 & 546 \\
\hline Escolaridad $\leq 8$ años $(\%)^{*}$ & 14,7 & 141 & 18,3 & 98 & 11,3 & 43 \\
\hline Sistema público de salud (\%)* & 66,1 & 740 & 73,2 & 441 & 59,5 & 299 \\
\hline Tabaquismo últimos 6 meses (\%)* & 51,5 & 620 & 46,1 & 302 & 58,1 & 318 \\
\hline Trabajo en mina o fundición (\%)* & 22,6 & 168 & 6,9 & 28 & 32,9 & 140 \\
\hline \multirow{2}{*}{$\begin{array}{l}\text { Características sociodemográficas } \\
\text { niños y adolescentes }\end{array}$} & \multicolumn{2}{|c|}{ Total $(n=193)$} & \multicolumn{2}{|c|}{ Mujeres $(n=93)$} & \multicolumn{2}{|c|}{ Hombres $(n=100)$} \\
\hline & Media o \% & $\mathbf{n}$ & Media o \% & $\mathbf{n}$ & Media o \% & $\mathbf{n}$ \\
\hline Edad, años (media) & 11,39 & 193 & 11,08 & 93 & 11,66 & 100 \\
\hline Escolaridad $\leq 8$ años (\%) & 68,8 & 135 & 70,6 & 67 & 65,9 & 68 \\
\hline Sistema público de salud (\%)* & 62,3 & 117 & 82,6 & 60 & 45,3 & 57 \\
\hline Tabaquismo por 6 meses o más (\%) & 4,1 & 8 & 6,4 & 6 & 2 & 2 \\
\hline
\end{tabular}

*Diferencias significativas por sexo $(p<0,05)$. 
Tabla 2. Distribución por precentiles (p 50, p75 y p95) de metales (oides) en adultos y niños habitantes de Antofagasta según sexo, año 2018

\begin{tabular}{|c|c|c|c|c|c|c|}
\hline & & & & & centil & \\
\hline & & & $\mathbf{N}$ & 50 & 75 & 95 \\
\hline Arsénico inorgánico $\mu \mathrm{g} / \mathrm{L}$ orina & Adultos & Total & 1.175 & 14,2 & 21,9 & 41,4 \\
\hline & & Mujeres & 631 & 12,9 & 21 & 36,5 \\
\hline & & Hombres & 539 & 14,7 & 22,2 & 45,9 \\
\hline & Niños & Total & 189 & 17,8 & 24,4 & 44,5 \\
\hline & & Mujeres & 91 & 12,8 & 24,3 & 44,9 \\
\hline & & Hombres & 98 & 19,8 & 23,9 & 43,8 \\
\hline Cadmio $\mu \mathrm{g} / \mathrm{L}$ orina & Adultos & Total & 1.189 & 0,6 & 1 & 1,8 \\
\hline & & Mujeres & 643 & 0,7 & 1 & 1,9 \\
\hline & & Hombres & 541 & 0,6 & 1 & 1,6 \\
\hline & Niños & Total & 191 & 0,5 & 0,6 & 0,9 \\
\hline & & Mujeres & 93 & 0,5 & 0,6 & 0,8 \\
\hline & & Hombres & 98 & 0,5 & 0,6 & 0,9 \\
\hline Mercurio $\mu \mathrm{g} / \mathrm{L}$ orina & Adultos & Total & 1.199 & 0,8 & 1,2 & 2,5 \\
\hline & & Mujeres & 652 & 0,8 & 1,2 & 2,8 \\
\hline & & Hombres & 542 & 0,8 & 1,2 & 2,1 \\
\hline & Niños & Total & 193 & 0,6 & 1 & 2 \\
\hline & & Mujeres & 93 & 0,5 & 0,8 & 1,2 \\
\hline & & Hombres & 100 & 0,7 & 1,4 & 2 \\
\hline Plomo $\mu \mathrm{g} / \mathrm{dL}$ sangre & Adultos & Total & 1.197 & 1,7 & 2,3 & 4,2 \\
\hline & & Mujeres & 647 & 1,5 & 2 & 3,6 \\
\hline & & Hombres & 545 & 1,9 & 2,7 & 4,4 \\
\hline & Niños & Total & 182 & 1,3 & 2 & 3,1 \\
\hline & & Mujeres & 89 & 1,3 & 1,6 & 2,9 \\
\hline & & Hombres & 93 & 1,1 & 2,2 & 3,1 \\
\hline Cromo $\mu \mathrm{g} / \mathrm{L}$ orina & Adultos & Total & 1.086 & 0,3 & 3,4 & 7,2 \\
\hline & & Mujeres & 589 & 0,3 & 2,8 & 7,4 \\
\hline & & Hombres & 493 & 0,3 & 4 & 6,6 \\
\hline & Niños & Total & 179 & 0,3 & 2,5 & 5,6 \\
\hline & & Mujeres & 86 & 0,3 & 2,4 & 5,5 \\
\hline & & Hombres & 93 & 0,3 & 2,5 & 5,6 \\
\hline
\end{tabular}

Para arsénico inorgánico urinario en adultos, las variables asociadas al aumento de los niveles fueron ser hombre $(\beta=3,12 \mu \mathrm{g} / \mathrm{L}$, IC $95 \% 0,19$ $-6,05)$ y vivir a más de 200 metros de los puntos de monitoreo realizados por las autoridades de salud o ambiente (Tabla 3). Mientras más lejos de estos puntos, mayor fue el incremento de AsIn $(\beta=9,63 \mu \mathrm{g} / \mathrm{L}$, IC 95\% 5,78-13,48). El tener un nivel educacional menor a 8 años de escolaridad resultó al borde de la significancia estadística. Las asociaciones que se presentaron respecto a los adultos, se ajustaron con variables tales como el trabajo en actividades de la minería o el consumo de pescado, estas últimas sin significancia estadística.

En el análisis de los resultados en niños, se observó que las variables asociadas a niveles aumentados de arsénico fueron: ingesta de pescados 


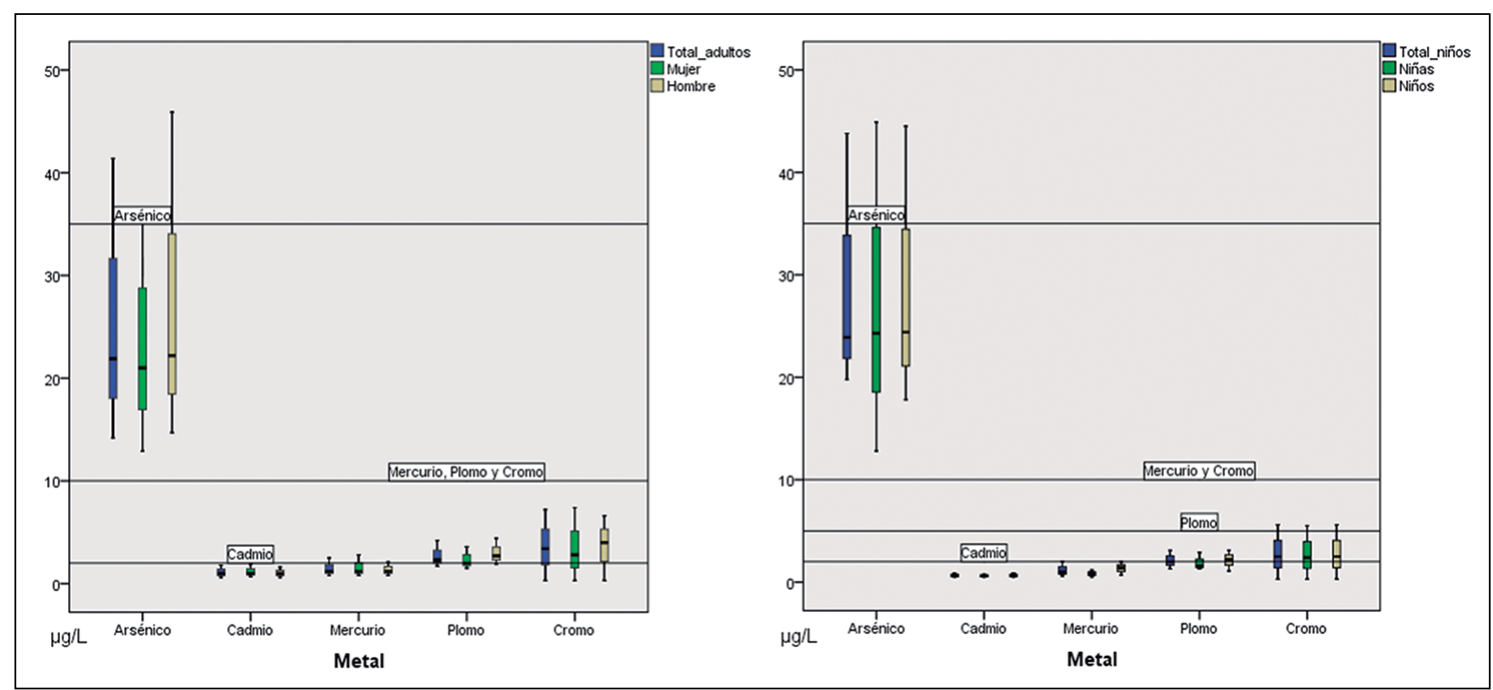

Figura 2. Concentración y distribución en percentiles de metales (oides) en adultos ( $n=1.203$ ) y niños $(n=193)$ habitantes de Antofagasta según sexo, año 2018. *Las líneas transversales representan el valor de referencia Minsal para cada metal: Arsénico $35 \mu \mathrm{g} / \mathrm{L}$, mercurio y cromo $10 \mu \mathrm{g} / \mathrm{L}$, plomo $10 \mu \mathrm{g} / \mathrm{dL}$ adultos y $5 \mu \mathrm{g} / \mathrm{dL}$ niños, cadmio $2 \mu \mathrm{g} / \mathrm{L}$.

Tabla 3. Modelo multivariado que indica asociaciones entre concentración de arsénico inorgánico en orina en adultos y otras variables de interés, año $2018(n=796)$

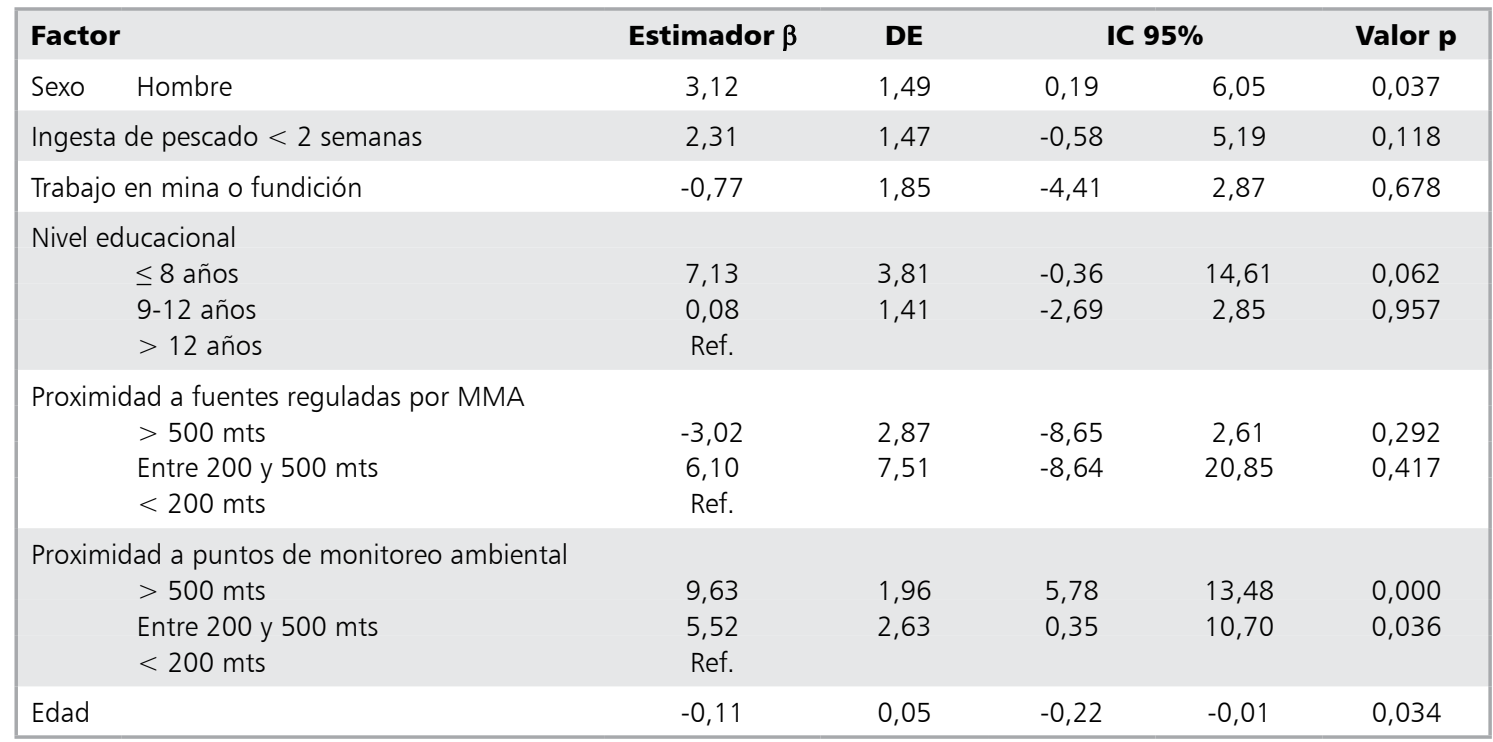

productos del mar $(\beta=9,11 \mu \mathrm{g} / \mathrm{L}$, IC $95 \% 4,14$ $-14,08)$ y tener un cuidador con menos de 8 años de escolaridad ( $\beta=9,82 \mu \mathrm{g} / \mathrm{L}$, IC 95\% 2,85 -16,79). La residencia a más de 500 metros del vertedero La Chimba también constituyó un factor de riesgo $(\beta=6,39 \mu \mathrm{g} / \mathrm{L}, \mathrm{IC} 95 \%$ 2,38 -10,40). Estos resultados deben evaluarse con precaución, dado que el bajo número de participantes en este subgrupo y las bajas concentraciones obtenidas, afectan la precisión de los modelos realizados. 


\section{Discusión}

En relación a la caracterización epidemiológica de la población de la comuna de Antofagasta, se observa un perfil parecido a lo señalado por estudios poblacionales, como las encuestas nacionales de salud. Destaca un mayor nivel de consumo de pescados, mariscos, y de tabaquismo, factores que pueden aumentar el riesgo de exposición a los metales estudiados o incrementar su efecto negativo ${ }^{4}$.

A pesar de que la autoridad sanitaria de la región encontró niveles elevados de metal/metaloides en material sedimentado de la ciudad, en este estudio, las personas expuestas presentan valores bajos, tomando como referencia los rangos sugeridos por el Ministerio de Salud ${ }^{3,5-8}$. La excepción es el arsénico inorgánico, que está sobre el valor referencial en $8 \%$ de la población adulta y en $12,3 \%$ en población infantil. Los valores de arsénico urinario encontrados en la población de Antofagasta no difieren mayormente a los encontrados en Chile previamente, desde el año 2000 hasta 2010, aunque cada estudio utilizó diferentes técnicas diagnósticas no necesariamente comparables ${ }^{9-12}$.

En la muestra general, destaca el predominio de ocupaciones de riesgo asociadas a la exposición a metales, tales como la minería. Sin embargo, la población que presentó niveles mayores de arsénico inorgánico, no tuvo significativamente mayor participación en este rubro. En relación a esto, se ha señalado que la población humana que no tienen exposición ocupacional, tiene como principal fuente la exposición a los alimentos y agua, lo que probablemente podría ser la fuente de contaminación en esta muestra ${ }^{3}$, aunque no se puede excluir como fuente de exposición el polvo generado por el tránsito de vehículos y el tabaquismo en grupos específicos.

Es de interés señalar que la mayor parte de la población reside en áreas que se encuentran a más de 500 metros de distancia de las fuentes de riesgo identificadas. Contrario a lo esperado, el mayor riesgo de presentar niveles alterados de los metales estudiados se produjo en esta población y crece al aumentar la distancia. Los hallazgos de este estudio sugieren que podrían existir otras fuentes de exposiciones ambientales más allá de los puntos de monitoreo ya evaluados, que deben ser pesquisadas y evaluadas a su contenido en metales y la cercanía con la población, de manera de reducir la exposición involuntaria a ellas.

Al establecer perfiles epidemiológicos de personas con valores de arsénico inorgánico alterado, se observó que aumenta en personas de sexo masculino, de baja escolaridad, que residen en áreas no controladas por la autoridad ambiental-salud. Entre las posibles explicaciones es que los hombres pudieran tener alguna actividad especial de riesgo, o que existan otras fuentes ambientales de las cuales no se dispone datos específicos. Este perfil es similar al de niños y adolescentes con niveles de arsénico inorgánico alterado. Estos aumentan en aquellos niños que reportaron mayor consumo de pescados y otros productos del mar, con apoderados de menos de 12 años de escolaridad, y que residen a más de 500 metros de La Chimba, lugar que pudiera tener algún riesgo de exposición no ponderado. Aquí toma relevancia explorar el impacto de la dieta en los niveles del metaloide en la población, puesto que la exposición a arsénico puede ocurrir a través del consumo de alimentos, como cereales (especialmente el arroz), productos lácteos, y agua no regulada, según reporta la literatura internacional ${ }^{13}$.

Dada la conocida asociación positiva entre niveles elevados de arsénico urinario y diabetes mellitus ${ }^{14,15}$, es importante planificar intervenciones dirigidas a prevención primaria específica en este tema. Por otra parte, se ha reportado una asociación entre niveles de arsénico urinario y la función pulmonar, encontrando patrones respiratorios restrictivos en niños con niveles elevados de arsénico y mayor riesgo de neumonía ${ }^{16}$. Este debería ser un punto de especial atención para la planificación sanitaria en prevención de enfermedades respiratorias y vacunación.

Se recomienda educar a la población de Antofagasta sobre la necesidad de reducir el consumo de tabaco en cualquiera de sus formas, dado de que se ha descrito que la interacción tabaquismo-arsénico puede tener un efecto sinérgico en el incremento del riesgo de cáncer ${ }^{17}$, lo que ya ha sido demostrado con evidencia epidemiológica en Antofagasta ${ }^{4}$.

Para personas que no exceden los valores referenciales, los esfuerzos se deben focalizar en estrategias de control de riesgo poblacional en grupos específicos, por ejemplo, en personas de menor nivel de escolaridad y nivel socioeconómi- 
co. Esto, asociado a la promoción de conductas de control y prevención.

En personas adultas que exceden valores referenciales, se recomienda apoyar los programas de vigilancia en salud ocupacional y fomentar programas antitabaco. En el caso del subgrupo de niños, se requiere establecer estudios específicos de medición de metales y efectos en salud, y será necesario, además, evaluar las condiciones de sus hogares y su entorno más cercano.

Se debe también investigar niveles ambientales de AsIn en todas las matrices ambientales que tienen contacto con las personas: aire, suelo, polvo en suspensión, alimentos. Es de especial interés evaluar áreas del territorio comunal que no hayan sido parte de monitoreos o vigilancias ambientales previas, así como fortalecer o desarrollar un sistema de vigilancia epidemiológico-ambiental que utilice herramientas geoespaciales en su análisis de información.

Cualesquiera sean las medidas a tomar por parte de la autoridad sanitaria, se recomienda repetir la determinación los indicadores poblacionales de AsIn con un plazo no mayor a 3 años y generar sistemas de vigilancia poblacionales centinela.

La principal limitación de este estudio es que no se dispone de estudios previos nacionales que aporten evidencia epidemiológica sobre prevalencia de exposición a metales en muestras con representatividad comunal, limitando la comparabilidad de estos resultados. Sin embargo, los valores obtenidos, especialmente al comparar medias o percentiles, no exceden lo establecido en otros estudios realizados en Chile.

Este estudio es el primero en su tipo en Chile por obtener una prevalencia con representatividad poblacional a nivel comunal. Se espera que sirva como referencia para los resultados que se obtengan una vez realizadas intervenciones orientadas al control de los principales metales/metaloides cuantificados en este estudio.

\section{Conclusión}

La residencia de personas en cercanía a fuentes fijas o móviles de contaminación a metales no se asocia a presencia de mayores niveles de estos en orina o sangre.

El arsénico inorgánico es un contaminante de importancia en la población de la comuna de
Antofagasta, por lo que se recomienda focalizar los esfuerzos para el control a nivel poblacional en este metal, identificando sus fuentes de exposición naturales y antropogénicas, fortaleciendo los sistemas de vigilancia de todas las matrices ambientales y evaluando marcadores de daño clínico y subclínico en personas con niveles alterados. Se recomienda evaluar el nivel exposición de arsénico después de realizar intervenciones sanitarias-ambientales, para establecer el impacto de las intervenciones recomendadas, en un plazo no mayor de 3 años.

Agradecimientos: Agradecemos al Gobierno Regional, la entrega de la base de datos solicitada a través de Transparencia, con fecha 31 de julio de 2019 ( $n^{\circ}$ solicitud AB077T0000509). Además, queremos agradecer a Mónica Cifuentes, enfermera coordinadora del proyecto y al equipo de salud de Antofagasta que participó en el trabajo de campo: Catalina Salinas, Katherine Troncoso, Patricia Plaza, Lilian Pérez, Claudia Cortés, Maria Elena Aguilera, Dagoberto Zamora, Viviana Monárdez, Eliana Rodríguez y Geraldy Barrientos. Finalmente, agradecer a: Ernesto Castillo, Ericka Ahumada, Patricia Berrios, Yerko Covacevich, Lucía Molina y Karina Juárez por sus valiosas labores ejecutadas en el desarrollo del estudio.

\section{Referencias}

1. Biblioteca del Congreso Nacional. Región de Antofagasta. Disponible en: https://www.bcn.cl/siit/nuestropais/ nuestropais/region2/ [Consultado el 4 de marzo de 2019].

2. Harris P, Taylor R, Thielke R, Payne J, González N, Jose G, et al. Research electronic data capture (REDCap) - A metadata-driven methodology and workflow process for providing translational research informatics support. J Biomed Inform 2009; 42 (2): 377-81.

3. Ministerio de Salud. Guía Clínica: Vigilancia Biológica de la Población Expuesta a Arsénico, Beneficiarios de la Ley 20.590. Santiago de Chile (2014). Disponible en: https://www.minsal.cl/sites/default/files/files/Guia_Clinica_Vigilancia_Arsenico_final.pdf [Consultado el 22 de marzo de 2019].

4. Ferreccio C, Yuan Y, Calle J, Benítez H, Parra RL, Acevedo J, et al. Arsenic, tobacco smoke, and occupation: associations of multiple agents with lung and bladder cancer. Epidemiology 2013b; 24 (6): 898-905. 
5. Ministerio de Salud. Guía Clínica: Vigilancia Biológica de la Población Expuesta a Cromo en la Comuna de Arica. Santiago de Chile (2014). Disponible en: https:// www.minsal.cl/sites/default/files/files/Guia_Clinica_Vigilancia_Cromo_final.pdf [Consultado el 22 de marzo 2019].

6. Ministerio de Salud. Guía Clínica: Vigilancia Biológica de la Población Expuesta a Plomo en la Comuna de Arica. Santiago de Chile, Chile (2014). Disponible en: https://www.minsal.cl/sites/default/files/files/Guia_Clinica_Vigilancia_Plomo_final.pdf [Consultado el 22 de marzo de 2019].

7. Ministerio de Salud. Guía Clínica Vigilancia Biológica de la Población Expuesta a Cadmio. Santiago de Chile (2014). Disponible en: https://www.minsal.cl/sites/default/files/files/Guia_Clinica_Vigilancia_Cadmio_final. pdf [Consultado el 22 de marzo de 2019].

8. Ministerio de Salud. Guía Clínica Vigilancia Biológica de la Población Expuesta a Mercurio en la Comuna de Arica. Santiago de Chile (2014). Disponible en: https:// www.minsal.cl/sites/default/files/files/Guia_Clinica_Vigilancia_Mercurio_final.pdf [Consultado el 22 de marzo de 2019].

9. Hopenhayn C, Ferreccio C, Browning R, Huang B, Peralta $\mathrm{C}$, Gibb $\mathrm{H}$, et al. Arsenic exposure from drinking water and birth weight. Epidemiology 2003; 14 (5): 593602.

10. Hopenhayn C, Huang B, Christian J, Peralta C, Ferreccio C, Atallah R, et al. Profile of urinary arsenic metabolites during pregnancy. Environ Health Perspect 2003; 111
(16): 1888-91.

11. Cáceres D, Pino P, Montesinos N, Atalah, Loomis D. Exposure to inorganic arsenic in drinking water and total urinary arsenic concentration in a Chilean population. Environ Res 2005; 98 (2): 151-5.

12. Ronco AM, Gutiérrez Y, Gras N, Muñoz L, Salazar G, Llanos MN. Lead and arsenic levels in women with different body mass composition. Biol Trace Elem Res 2010; 136 (3): 269-78.

13. Gundert-Remy, Ursula. High exposure to inorganic arsenic by food: the need for risk reduction. Archives of Toxicology 2015; 89 (12): 2219-27.

14. Feseke S, St-Laurent J, Anassour-Sidi E, Ayotte P, Bouchard M, Levallois P. Arsenic exposure and type 2 diabetes: results from the Canadian Health Measures Survey 2007. Health Promot Chronic Dis Prev Can 2007; 35 (4): 63-72.

15. Kuo C, Weaver V, Fadrowski J, Lin Y, Guallar E, Navas-Acien A. Arsenic exposure, hyperuricemia, and gout in US adults. Environment International 2015; 76 : $32-40$.

16. George C, Brooks W, Graziano J, Nonyane B, Hossain L, Goswami D, et al. Arsenic exposure is associated with pediatric pneumonia in rural Bangladesh: a case control study. Environ Health 2015; 23: 14: 83.

17. Prasenjit R, Mukherjee A, Giri S. Evaluation of genetic damage in tobacco and arsenic exposed population of Southern Assam, India using buccal cytome assay and comet assay. Ecotoxicology and Environmental Safety 2016; 124: 169-76. 